\title{
Universality and Scaling in Relations Between the Plasmon Energy and Solid-State Parameters: Viewing Nanoscale Properties of Battery Materials
}

Vladimir Oleshko

NIST, Gaithersburg, Maryland, United States

Plasmons generated by fast electrons passing through solids are the major electron excitations in the lowloss energy range. The energy of volume plasmons is related to the valence electron density $n$ as $E_{p} \cong$ $\left[\left(\hbar \omega_{p}\right)^{\mathrm{f}}+E_{g}\right]^{0.5}$, where $\omega_{\mathrm{p}}^{\mathrm{f}}=\hbar\left(n \mathrm{e}^{2} / \varepsilon_{0} \mathrm{~m}\right)^{0.5}$ and $E_{\mathrm{gis}}$ the bandgap. Valence electron densities govern many intrinsic solid-state parameters of materials, e.g., elastic moduli, hardness, cohesive energy, thermal and electrical conductivities, etc. Insights into $E_{p}$-material property relations offer opportunities for probing and tailoring multiple properties of nanostructured materials to realize their ultimate performance [1]. Herein we extend universality and scaling on mechanical, cohesive, and electrochemical properties of materials for energy storage (ES) composed of Group I-IV elements. Fundamental periodic trends and factors influencing gravimetric $\left(C_{g}\right)$ and volumetric $\left(C_{v}\right)$ capacities and energy density of ES materials are considered in the relation to mechanical and cohesive properties. The origin of connections between the material properties and $E_{p}$ lies in the nature of electron-ion interactions and essentially exponential decay of electron density with interatomic distance. For materials with metallic and preferentially covalent bonding, this is established by the universal binding energy relation (UBER), which describes the shape of the binding energy curve for cohesion in solids, interfaces, chemisorption on metal surfaces, and bonding $[2,3]$. In the UBER, the bulk modulus, $B_{m}$, is related to $E_{c o h}$ and the equilibrium Wigner-Seitz (WS) atomic radius, $r_{w s e}$, via the dimensionless anharmonicity parameter $\eta=r_{w s e} l$, as $B_{m}=(1 / 12 \pi) r_{w s e}$ ${ }^{3} E_{c o h} \eta^{2}=\left(\varepsilon_{0} \mathrm{~m} / 9 \mathrm{e}^{2} \hbar^{2} N_{v e}\right) E_{c o h} \eta^{2}\left(E_{p}{ }^{2}-E_{g}{ }^{2}\right)(1)$, where $l$ is the characteristic length describing the width of the binding energy curve or the range over which strong forces act that sets the range of the Hooke's-law region [2], $N_{v e}$ is the number of valence electrons per atom. From Eqn. (1), $B_{m} \propto E_{c o h} / V_{w s e} \propto E_{p}{ }^{2}-E_{g}{ }^{2}$, where $\mathrm{V}_{\mathrm{wse}}$ is the volume of the WS cell at equilibrium. From linear regression analyses of experimental data and DFT calculations (Figs. 1a and 1b, 2a), the relations can be generalized using an equation of the type $P_{m}=\mathrm{A}\left(E_{p}{ }^{2}-E_{g}{ }^{2}\right)^{\mathrm{B}}$, where $P_{m}=C_{g}, C_{v}, B_{m}$, or $E_{c o h} / V_{w s e}$, and $A$ and $B$ are $l s q$-fitted structure-dependent parameters. For $B_{m}$, and $E_{c o h} / V_{w s e}$, scaling can be deduced from Eqn. (1) since the valence electron density governs variations of both $B_{m}$ and $E_{c o h} / V_{w s e}$ with $E_{p}$. Universality and scaling in $E_{p}$ property relations demonstrate that $E_{p}$ is an invaluable parameter to investigate the nanoscale properties of engineering ES materials. Fig. 2 illustrates the capabilities of the technique utilized for spatially-resolved quantitative plasmon-ratio mapping of microhardness $H_{m}$ in a sulfur copolymer - carbon composite cathode for Li-S batteries. The derived relations can be employed to determine and visualize in situ nanoscale properties of ES materials using low-loss STEM-EEL spectroscopic imaging. 


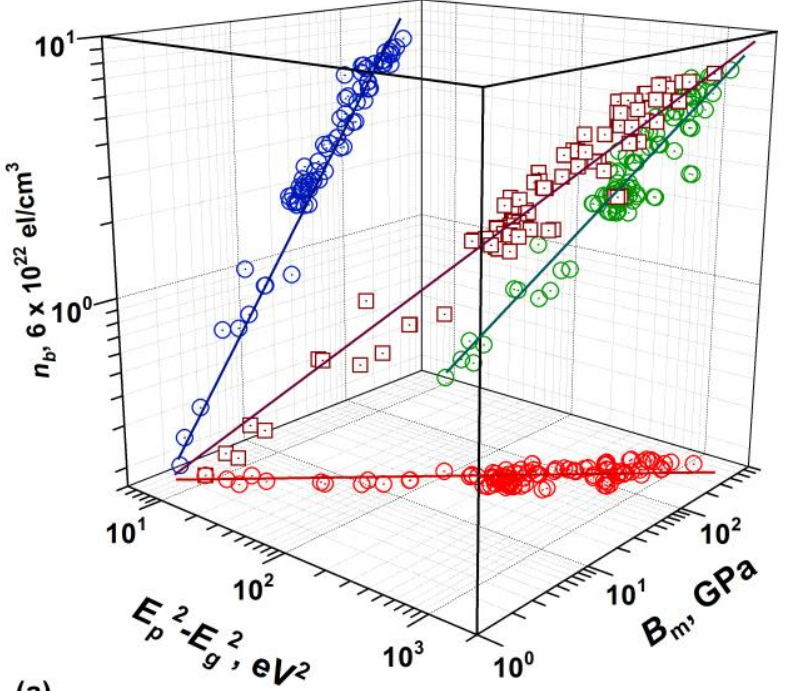

(a)

Figure 1. Figure 1. (a) 3D lsq-fitted correlations between the electron density $n$ at the boundary of the WS cell, the Bm, and Ep2-Eg2 for 58 metals, B, C, Si, and Ge [1]. (b) Correlations between gravimetric capacity Cg and Ep2-Eg2 for Group I-IV elements.
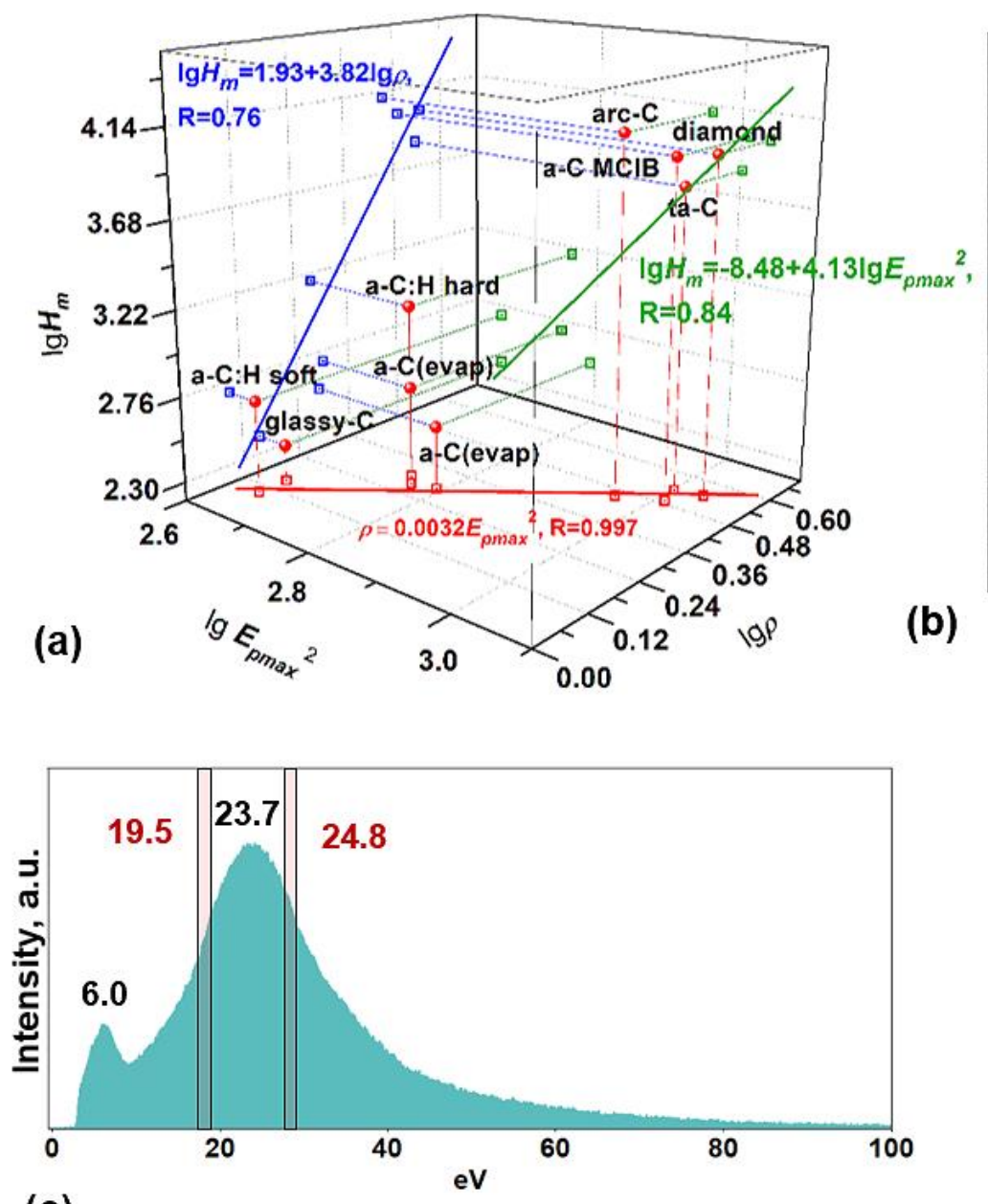

(c)

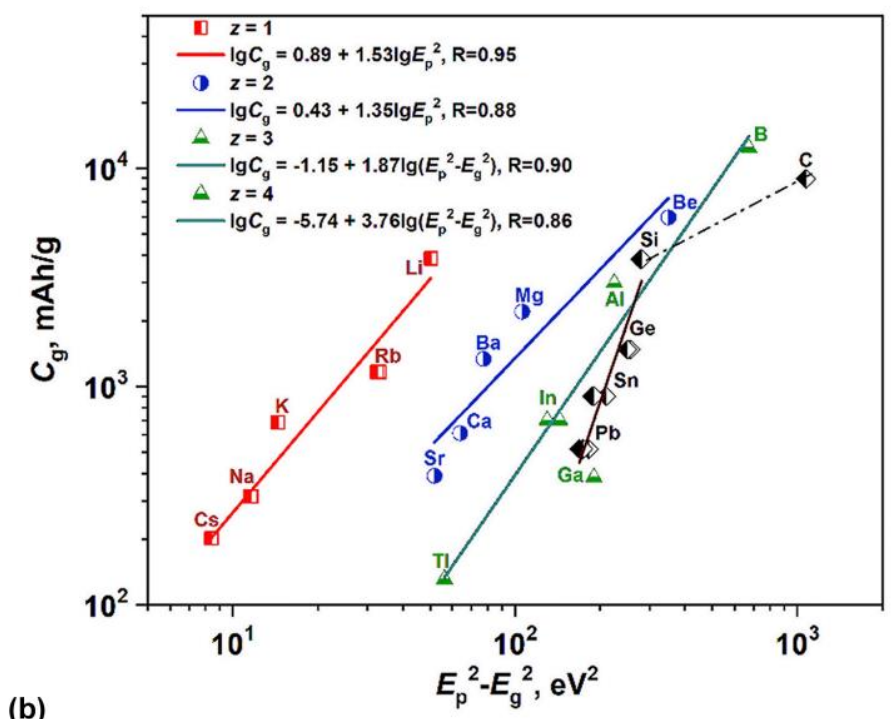


Figure 2. Figure 2. (a) 3D lsq-fitted correlations between the microhardness Hm, density $\rho$, and Epmax for carbon materials [1]. (b) HAADF STEM, thin section of a sulfur copolymer-carbon cathode. (c) Lowloss EEL spectrum from the red square in (b), single scattering distribution with $0.3 \mathrm{eV}$ windows at 19.5 $\mathrm{eV}$ and $24.8 \mathrm{eV}$. (d) Plasmon-ratio $\mathrm{Hm}$ map derived from an area marked by the green rectangle in (b).

\section{References}

[1] V.P. Oleshko, J. Nanosci. Nanotechnol. 12 (2012) 8580-8588.

[2] J. Rose, et al, Phys. Rev. B29 (1984) 2963.

[3] J Li, et al, J Appl. Phys. 113, (2013) 023516. 